\title{
Small-Angle Neutron Scattering on a Core-Shell Colloidal System: A Contrast-Variation Study
}

\author{
M. Zackrisson, ${ }^{*}$, A. Stradner,$\stackrel{\dagger}{\dagger}$ P. Schurtenberger,$\stackrel{\dagger}{\dagger}$ and J. Bergenholtz ${ }^{\dagger}$ \\ Department of Chemistry, Göteborg University, SE-412 96, Göteborg, Sweden, and \\ Department of Physics, University of Fribourg, CH-1700, Fribourg, Switzerland
}

\begin{abstract}
Small-angle neutron scattering (SANS) measurements are reported on a sterically stabilized, coreshell colloidal system using contrast variation. Aqueous dispersions of polystyrene particles bearing grafted poly(ethylene glycol) (PEG) have been studied over a large range of particle concentrations and two different solvent conditions for the PEG polymer. SANS data are analyzed quantitatively by modeling the particles as core-shell colloids. In a good solvent and under particle contrast conditions, an effective hard-sphere interaction captures excluded-volume interactions up to high concentrations. Contrast variation, through isotopic substitution of both the core and solvent, expedite a detailed study of the PEG layer, both in the dilute limit and as a function of the particle concentration. Upon diminishing the solvent quality, subtle changes in the PEG layer translate into attractions among particles of moderate magnitude.
\end{abstract}

\section{Introduction}

Aqueous dispersions of colloids find uses in a wide variety of technological applications, including paints, adhesives, inks, and coatings. Such applications rely on the dispersions being stable over a range of physicochemical conditions. Polymeric surface modification of colloidal particles, particularly through polymer grafting, is one of the more robust means for imparting stability to colloidal systems. ${ }^{1,2}$ Poly(ethylene glycol) (PEG) is a polymer wellsuited to this task and is widely used for stabilizing aqueous dispersions of solid particles, ${ }^{3-11}$ as well as for the in vivo protection of liposomes ${ }^{12,13}$ and for the modification of proteins. ${ }^{14-16}$ Also, ethylene-oxide-based

* To whom correspondence should be addressed. Telephone: +46 31-772-2815. Fax: +46-31-772-1394.E-mail: malinz@chem.gu.se.

Göteborg University.

$¥$ University of Fribourg.

(1) Napper, D. H. Polymeric Stabilization of Colloidal Dispersions; Academic Press: London, U.K., 1983.

(2) Russel, W. B.; Saville, D. A.; Schowalter, W. R. Colloidal Dispersions; Cambridge University Press: New York, 1989.

(3) Ottewill, R. H.; Satgurunathan, R. Colloid Polym. Sci. 1987, 265, 845.

(4) Ploehn, H. J.; Goodwin, J. W. Chemical grafting of poly(ethylene oxide) onto polystyrene latex. In Polymers as Rheology Modifiers; Schultz, D. N., Glass, J. E., Eds.; ACS Symposium Series 462: Washington, DC, 1991 .

(5) Maste, M. C. L.; van Velthoven, A. P. C. M.; Norde, W.; Lyklema, J. Colloids Surf., A 1994, 83, 255.

(6) Vidal, F.; Guyot, A. New J. Chem. 1995, 19, 1081.

(7) Brindley, A.; Davis, S. S.; Davies, M. C.; Watts, J. F. J. Colloid Interface Sci. 1995, 171, 150.

(8) Furuhashi, H.; Kawaguchi, S.; Itsuno, S.; Ito, K. Colloid Polym. Sci. 1997, 275, 227.

(9) Weiss, A.; Hartenstein, M.; Dingenouts, N.; Ballauff, M. Colloid Polym. Sci. 1998, 276, 794.

(10) Shay, J. S.; English, R. J.; Spontak, R. J.; Balik, C. M.; Khan, S. A. Macromolecules 2000, 33, 6664.

(11) Meng, F.; Engbers, G. H. M.; Feijen, J. J. Biomed. Mater. Res. A 2004, 70, 49 .

(12) Klibanov, A. L.; Maruyama, K.; Torchilin, V. P.; Huang, L. FEBS Lett. 1990, 268, 235.

(13) Allen, T. M.; Hansen, C.; Martin, F.; Redemann, C.; Yauyoung, A. Biochim. Biophys. ACTA 1991, 1066, 29.

(14) Abuchowski, A.; Vanes, T.; Palczuk, N. C.; Davis, F. F. J. Biol. Chem. 1977, 252, 3578.

(15) Lundblad, R. L.; Bradshaw, R. A. Biotech. Appl. Biochem. 1997, $26,143$.

(16) Caliceti, P.; Veronese, F. M. Adv. Drug Delivery Rev. 2003, 55, 1261. surfactants and copolymer blocks are used widely in forming colloidal size complexes and self-assembled structures. An added incentive for turning to stabilization by PEG comes from its biocompatibility and the prolonged circulatory times exhibited in biomedical imaging ${ }^{17}$ and drug delivery ${ }^{18-23}$ applications of PEG-modified colloids.

From a more fundamental standpoint, the interactions among PEG-grafted colloids follow qualitatively the aqueous solubility of $\mathrm{PEG}$, such that they can readily be tuned, from repulsive under good solvent conditions to attractive under marginal solvent conditions, in a fully reversible fashion. It follows that such systems can become a platform for systematic studies of colloidal interactions on equilibrium structural and dynamic properties, as well as properties out of equilibrium.

Polymer-grafted colloidal spheres obtain a core-shell structure, comprising concentric domains of two chemically different materials, a structure that is ubiquitous in the colloid science field. While in this case it is produced by design, similar structures result also from the natural tendency for microscale segregation, as in the spontaneous self-assembly of droplet microemulsions or block copolymer micelles. Structural information on core-shell-like systems can be accessed very effectively with scattering techniques. In particular, they can be studied in great detail using contrast-variation methods, ${ }^{24}$ achieved most readily in the context of small-angle neutron scattering (SANS), although several studies make use of light ${ }^{25-27}$ or X-ray ${ }^{28-30}$ scattering.

(17) Torchilin, V. P. Adv. Drug Delivery Rev. 2002, 54, 235.

(18) Gref, R.; Minamitake, Y.; Peracchia, M. T.; Trubetskoy, V.; Torchilin, V.; Langer, R. Science 1994, 263, 1600.

(19) Dunn, S. E.; Brindley, A.; Davis, S. S.; Davies, M. C.; Illum, L. Pharm. Res. 1994, 11, 1016.

(20) Storm, G.; Belliot, S. O.; Daemen, T.; Lasic, D. D. Adv. Drug Delivery Rev. 1995, 17, 31.

(21) Brigger, I.; Morizet, J.; Aubert, G.; H. Chacun, M.-J. T.-L.; Couvreur, P.; Vassal, G. J. Pharmacol. Exp. Ther. 2002, 303, 928.

(22) Brigger, I.; Dubernet, C.; Couvreur, P. Adv. Drug Delivery Rev. $2002,54,631$.

(23) Allen, T. M.; Cullis, P. R. Science 2004, 303, 1818.

(24) Schurtenberger, P. Contrast and contrast variation in neutron, X-ray, and light scattering. In Neutrons, $X$-rays and Light: Scattering Methods Applied to Soft Condensed Matter; Lindner, P., Zemb, T., Eds.; North-Holland: Amsterdam, The Netherlands, 2002. 
Through contrast variation, via the variation of scattering contrast by selective isotopic substitution of protons for deuterium in SANS, small-scale structures can be discriminated. This has been amply demonstrated in the past for, e.g., polymer-coated particles, ${ }^{31-33}$ self-assembled surfactant ${ }^{34-37}$ and block copolymer ${ }^{38-41}$ systems. In addition, the quantitative modeling of SANS data for the same system under variable contrast is a far more stringent test of the models used and can therefore deliver more reliable values for structural parameters. As an alternative, combining modeling of, e.g., small-angle neutron and X-ray scattering, which generally derive from very different contrast profiles, is another way of obtaining a more exacting analysis. ${ }^{42,43}$ In passing, we note that it is considerably more difficult to make full use of contrastvariation methods for dispersions of solid-core particles, simply because of the inability to adjust the isotopic composition of the particle cores, which generally requires a separate synthesis, and the difficulty in exchanging solvent.

In this work, we use SANS to investigate aqueous dispersions of PEG-grafted polystyrene spheres over a wide range of particle concentrations, both under good and marginal solvent conditions. In particular, we focus on how positional correlations develop as a function of increasing particle concentration and how they are modified on going from good to marginal solvent conditions. In addition, we use contrast variation to focus on the properties of the PEG layer and how it responds to changes in the particle concentration and solvent conditions as modulated by addition of $\mathrm{Na}_{2} \mathrm{CO}_{3}$. Under good solvent conditions, particle correlations are well-captured up to high concentrations by an effective hard-sphere model, whereas under marginal solvent conditions, modeling the scattering from moderately concentrated dispersions requires adding attractions among particles.

In what follows, we first describe the preparation and characterization of the systems studied, as well as the conducted experiments. The theoretical basis for the models used in the quantitative fitting of the SANS data

(25) Ricka, J.; Borkovec, M.; Hofmeier, U. J. Chem. Phys. 1991, 94 8503.

(26) Christ, S.; Schurtenberger, P. J. Phys. Chem. 1994, 98, 12708.

(27) Bryant, G.; Mortensen, T.; Henderson, S.; Williams, S.; van Megen, W. J. Colloid Interface Sci. 1999, 216, 401.

(28) Dingenouts, N.; Ballauff, M. Acta Polym. 1993, 44, 178.

(29) Dingenouts, N.; Kim, Y. S.; Ballauff, M. Colloid Polym. Sci. 1994, 272,1380 .

(30) Dingenouts, N.; Bolze, J.; Pötschke, D.; Ballauff, M.Adv. Polym. Sci. 1999, 144, 1 .

(31) Ottewill, R. H.; Cole, S. J.; Waters, J. A. Macromol. Symp. 1995 92,97

(32) Dingenouts, N.; Seelenmeyer, S.; Deike, I.; Rosenfeldt, S.; Ballauff, M.; Lindner, P.; Narayanan, T. Phys. Chem. Chem. Phys. 2001, 7, 1169.

(33) Hone, J. H. E.; Cosgrove, T.; Saphiannikova, M.; Obey, T. M.; Marshall, J. C.; Crowley, T. L. Langmuir 2002, 18, 855.

(34) Kotlarchyk, M.; Huang, J. S.; Chen, S.-H. J. Phys. Chem. 1985 89,4382 .

(35) Eastoe, J.; Dong, J.; Hetherington, K. J.; Steytler, D.; Heenan,

R. K. J. Chem. Soc., Faraday Trans. 1996, 92, 65.

(36) Bagger-Jörgensen, H.; Olsson, U.; Mortensen, K. Langmuir 1997 13, 1413 .

(37) Bumajdad, A.; Eastoe, J.; Heenan, R. K.; Lu, J. R.; Steytler, D.

C.; Egelhaaf, S. J. Chem. Soc., Faraday Trans. 1998, 94, 2143.

(38) McConnell, G. A.; Lin, E. K.; Gast, A. P.; Huang, J. S.; Lin, M.

Y.; Smith, S. D. Faraday Discuss. 1994, 98, 121.

(39) Förster, S.; Wenz, E.; Lindner, P. Phys. Rev. Lett. 1996, 77, 95.

(40) Mortensen, K. J. Phys.: Condens. Matter 1996, 8, A103.

(41) Willner, L.; Poppe, A.; Allgaier, J.; Monkenbusch, M.; Lindner, P.; Richter, D. Europhys. Lett. 2000, 51, 628.

(42) Seelenmeyer, S.; Deike, I.; Rosenfeldt, S.; Norhausen, Ch.; Dingenouts, N.; Ballauff, M.; Narayanan, T.; Lindner, P. J. Chem. Phys. 2001, 114, 10471.

(43) Sommer, C.; Pedersen, J. S.; Garamus, V. M. Langmuir 2005 $21,2137$. is then set out. Subsequently, we report results extracted from the SANS modeling of systems examined under good solvent conditions, followed by results on the same systems exposed to marginal solvent conditions. Here, subtle structural changes of the PEG layer results in attractive interactions among colloids, in addition to the repulsive, excluded-volume interaction observed under good solvent conditions.

\section{Theory}

In elastic small-angle neutron scattering experiments, the measured quantity of interest is the coherent differential scattering cross-section $I(q)$ as a function of the magnitude of the scattering vector $q$, defined as $q=(4 \pi /$ $\lambda) \sin \theta / 2$, with $\lambda$ being the neutron wavelength and $\theta$ being the scattering angle. Synthetic colloids are always more or less polydisperse in size, and effects of polydispersity on $I(q)$ are readily included by treating the system as a discrete mixture of $N$ differently sized species. ${ }^{44,45}$ As described in detail in Appendix A, we impose a triangular size distribution, skewed such that few large particles are present. For a mixture, $I(q)$ is defined as

$$
I(q)=n \sum_{i, j=1}^{N}\left(x_{i} x_{j}\right)^{1 / 2} f_{i}(q) f_{j}^{*}(q) S_{i j}(q)
$$

where $x_{i}$ are mole fractions, $f_{i}(q)$ are form amplitudes (* denotes complex conjugation), $S_{i j}$ are partial structure factors, and the sum runs over the $N$ particle species in the mixture. In the noninteracting, dilute limit, $S_{i j}=\delta_{i j}$, leaving

$$
I(q)=n \sum_{i=1}^{N} x_{i}\left|f_{i}(q)\right|^{2}
$$

which is used to identify $P(q)$, the so-called form factor, viz $I(q)=n P(q)$. Strictly speaking, in this case, it is the "measured" form factor of the mixture; for monodisperse systems $(N=1)$, the sum vanishes and $P(q)$ reduces to the square of the form amplitude. The form factor contains information on the particle size, shape, and internal morphology, making it important to determine with as much detail and as few significant, independent parameters as possible.

We assume that a radially symmetric, core-shell structure mimics the distribution of nuclei in the particles sufficiently well. However, in place of a core-shell, stepfunction contrast profile, we attempt to capture, in the simplest possible way, the role of curvature. Assuming that interchain interactions cause PEG to adopt stretched conformations, we take the polymer molecules as identical rigid cylinders attached to the particle surfaces, pointing radially outward into the solvent. For this pincushion model, the local polymer volume fraction is

$$
\phi_{\mathrm{p}}(r) \approx \frac{N_{\mathrm{p}}}{4}\left(\frac{r_{0}}{r}\right)^{2}
$$

where $N_{\mathrm{p}}$ is the number of polymers per particle, $r_{0}$ is the radius of the circular cross-section of a polymer, and $r$ is the radial distance from the particle center. The volume fraction of the polymer in the solvated polymer layer is given as

(44) D’Aguanno, B.; Klein, R. Phys. Rev. A 1992, 46, 7652

(45) Klein, R.; D'Aguanno, B. Static scattering properties of colloidal suspensions. In Light Scattering, Principles, and Development; Brown, W., Ed.; Oxford University Press: London, U.K., 1996. 


$$
\phi_{\mathrm{p}}=\frac{4 \pi \int_{a}^{a+\delta} d r r^{2} \phi_{\mathrm{p}}(r)}{4 \pi \int_{a}^{a+\delta} d r r^{2}}=\frac{3 N_{\mathrm{p}}}{4}\left(\frac{r_{0}}{a}\right)^{2} \frac{\lambda-1}{\lambda^{3}-1}
$$

with $\delta$ being the layer thickness, $a$ being the core radius, and $\lambda=1+\delta / a$. Noting that the grafting density is $\sigma=$ $N_{\mathrm{p}} / 4 \pi a^{2}$, the area coverage is $A=\pi r_{0}^{2} \sigma$, and the local polymer distribution can be expressed as $\phi_{p}(r)=A(a / r)^{2}$. The parameter $A$ is the fraction of the sphere area occupied by the polymer, which is proportional to the grafting density.

In assembling a scattering length density profile, we supplement the polymer profile with a homogeneous core of radius $a$, giving the following

$$
\rho(r)-\rho_{\text {solv }}= \begin{cases}\rho_{\text {core }}-\rho_{\text {solv }} & 0<r<a \\ \left(\rho_{\text {peg }}-\rho_{\text {solv }}\right) \phi(r) & a<r<a \\ 0 & a+\delta<r\end{cases}
$$

where $\rho_{\text {shell }}=\phi_{p}(r) \rho_{\text {peg }}+\left(1-\phi_{p}(r)\right) \rho_{\text {solv }}$ has been used and $\rho_{\text {core }}, \rho_{\text {shell }}, \rho_{\text {peg }}$, and $\rho_{\text {solv }}$ are scattering length densities of the core, shell, polymer, and solvent, respectively. We have used $\rho_{\text {peg }}=6.37 \times 10^{-5} \mathrm{~nm}^{-2}{ }^{46}$ The form amplitude for this core-shell model is determined as

$$
\begin{aligned}
f(q)= & 4 \pi \int_{0}^{\infty} d r r^{2}\left(\rho(r)-\rho_{\text {solv }}\right) \frac{\sin (q r)}{q r} \\
= & 3 v\left\{\left(\rho_{\text {core }}-\rho_{\text {solv }}\right) \frac{j_{1}(q a)}{q a}+\right. \\
& \left.A\left(\rho_{\text {peg }}-\rho_{\text {solv }}\right) \frac{\operatorname{Si}(q \lambda a)-\operatorname{Si}(q a)}{q a}\right\}
\end{aligned}
$$

where $j_{1}(x)=x^{-2}(\sin (x)-x \cos (x))$ is the first-order spherical Bessel function, $\operatorname{Si}(x)=\int_{0}^{x} d t t^{-1} \sin (t)$ is the sine integral, and $v=4 \pi a^{3} / 3$ is the volume of the homogeneous core. In generalizing eq 6 to an $N$-component mixture, the grafting density is taken to be independent of the particle size. In addition, we assume the polymer layer thickness $\delta$ to be constant because the commercial macromonomer that we use is of low polydispersity, leading to the following final expression for the form amplitudes

$$
\begin{array}{r}
f_{i}(q)=3 v_{i}\left\{\left(\rho_{\text {core }}-\rho_{\text {solv }}\right) \frac{j_{1}\left(q a_{i}\right)}{q a_{i}}+\right. \\
\left.A\left(\rho_{\text {peg }}-\rho_{\text {solv }}\right) \frac{S i\left(q \lambda_{i} a_{i}\right)-S i\left(q a_{i}\right)}{q a_{i}}\right\}
\end{array}
$$

where $\lambda_{i}(q)=1+\delta / a_{i}$ and $v_{i}=4 \pi a_{i}^{3} / 3$. To model the partial structure factors $S_{i j}(q)$, we make use of the analytical Percus-Yevick solution for hard-sphere mixtures, first obtained by Lebowitz, ${ }^{47}$ as formulated by Blum and Stell. ${ }^{48,49}$ They succeeded in analytically inverting the matrix of direct correlation functions, and as a consequence, a numerical inversion step in determining $S_{i j}(q)$ is conveniently avoided. In adopting an effective hardsphere interaction, we require a way to compensate for the fact that the particles in reality do not act as true hard spheres. In addition, we wish to couple the form and structure factors via the size distribution without introducing too many additional parameters. To this end, we

(46) Won, Y.-Y.; Davis, H. T.; Bates, F. S.; Agamalian, M.; Wignall, G. D. J. Phys. Chem. B. 2000, 104, 7134.

(47) Lebowitz, J. L. J. Phys. Rev. 1964, 133, 895.

(48) Blum, L.; Stell, G. J. Chem. Phys. 1979, 71, 42. 2200 .

(49) Griffith, W. L.; Triolo, R.; Compere, A. L. Phys. Rev. A 1987, 35,
Table 1. Masses and Volumes Used in Syntheses of Latices $\mathrm{H8}$ and D8

\begin{tabular}{ccccccc}
\hline & $\begin{array}{c}\mathrm{H} 8 \\
\text { styrene } \\
(\mathrm{g})\end{array}$ & $\begin{array}{c}\mathrm{D} 8 \\
\text { styrene } \\
(\mathrm{g})\end{array}$ & $\begin{array}{c}\text { mPEG } \\
\text { acrylate } \\
(\mathrm{g})\end{array}$ & $\begin{array}{c}\mathrm{H}_{2} \mathrm{O} \\
(\mathrm{L})\end{array}$ & $\begin{array}{c}\mathrm{D}_{2} \mathrm{O} \\
(\mathrm{L})\end{array}$ & $\begin{array}{c}\text { KPS } \\
\text { initiator } \\
(\mathrm{g})\end{array}$ \\
\hline H8 latex & 11.4 & & 8.32 & 2.0 & & 0.100 \\
D8 latex & 0.1513 & 5.0 & 3.76 & & 0.9 & 0.045
\end{tabular}

define an effective hard-sphere radius by assuming that it is proportional to the actual core-shell radius, as $a_{i}^{\mathrm{HS}}$ $=\left(a_{i}+\delta\right) \alpha$, where $\alpha$ is a factor that permits for inflating or contracting the interaction radii. The partial structure factors are fully specified by the number densities $n_{i}=n x_{i}$ and radii $a_{i}^{\mathrm{HS}}$; a hard-sphere volume fraction is then determined self-consistently as $\phi^{\mathrm{HS}}=\Sigma_{i}^{N} 4 \pi n_{i}\left(a_{i}^{\mathrm{HS}}\right)^{3} / 3$. Note that the inclusion of structure factor effects in this way only introduces one extra parameter, $\alpha$. We note that, while the Percus-Yevick approximation is very accurate up to volume fractions $\sim 0.45$ for monodisperse hard spheres, it appears to be accurate over a larger range of volume fractions for polydisperse hard spheres. ${ }^{50}$

In summary, the input parameters to the $I(q)$ model are the mean core radius, $\bar{a}$, the polydispersity, $\sigma_{a} / \bar{a}$, the total number density, $n$, the thickness of the polymer layer, $\delta$, the surface coverage of the polymer, $A$, and the effective hard sphere radius, i.e., $\alpha$.

\section{Experimental Procedures}

A convenient route to obtaining core-shell particles with a grafted layer on a solidlike core is by copolymerizing styrene together with a macromonomer, $3,8,51$ where the macromonomer, having a more or less surfactant-like character, partitions mainly at the surfaces of the particles. ${ }^{5,52}$ In this work, we synthesize sterically stabilized nanoparticles by copolymerizing methyl poly(ethylene glycol) acrylate (mPEG acrylate) and styrene, via radical polymerization, essentially following the procedure devised by Brindley et al. ${ }^{7}$ Two latex formulations of different core isotopic compositions were synthesized, one using H8 styrene and the other composed of a monomer mixture of $\mathrm{H} 8$ and D8 styrene, with the former labeled as latex H8 and the latter as latex D8. The amounts and volumes of reactants and solvents are listed in Table 1.

Deuteration of the styrene core was needed to increase the scattering length density close to that of $\mathrm{D}_{2} \mathrm{O}$. In the preparation of latex D8, contact with water was avoided by synthesizing and purifying entirely in $\mathrm{D}_{2} \mathrm{O}$. The resulting $\mathrm{D} 8$ latex consisted of cores with a 95:5 molar ratio (assuming full conversion) of D8/ $\mathrm{H} 8$ polystyrene and the $\mathrm{H} 8$ latex of $\mathrm{H} 8$ polystyrene cores, with both latices surrounded by a polymer layer of end-grafted PEG. The small amount of $\mathrm{H} 8$ styrene added in the synthesis of latex D8 served to ensure that the core scattering length density would not exceed that of pure $\mathrm{D}_{2} \mathrm{O}$. The macromonomer, mPEG acrylate, with a molecular weight $\left(M_{\mathrm{w}}\right)$ of $2000 \mathrm{~g} / \mathrm{mol}$, was obtained from SunBio (South Korea) and used without further purification. The initiator potassium persulfate (KPS, 99.99\%) along with sodium carbonate $(99.5 \%)$, from Sigma-Aldrich and Aldrich, were used as received. $\mathrm{D}_{2} \mathrm{O}$ (99\%) was purchased from Cambridge Isotope Laboratories. Deuterated styrene (98\%), also from Cambridge Isotope Laboratories, together with styrene (99.5\%), purchased from Fluka, were washed with aluminum oxide prior to use to remove the stabilizing agent. The macromonomer and initiator were added by weight and dissolved in Milli- $\mathrm{Q}$ water or $\mathrm{D}_{2} \mathrm{O}$ for latex $\mathrm{D} 8$ and added to the reaction flask at $70{ }^{\circ} \mathrm{C}$ together with styrene, added beneath the liquid surface. During synthesis, in a $2 \mathrm{~L}$ four-necked, round-bottomed flask, a constant stirring rate of $300 \mathrm{rpm}$ for the large-volume synthesis and 200 rpm for the D8 latex synthesis was kept and the reaction

(50) Frenkel, D.; Vos, R. J.; de Kruif, C. G.; Vrij, A. J. Chem. Phys. 1986, 84, 4625 .

(51) Capek, I. Adv. Colloid Interface Sci. 2000, 88, 295.

(52) Brindley, A.; Davies, M. C.; Lynn, R. A. P.; Davis, S. S.; Hearn, J.; Watts, J. F. Polymer 1992, 33, 1112. 
Table 2. Complete Set of Parameter Values Obtained from Fits of the Model to the Concentration Series of Latex H8, Which Corresponds to the Solid Lines in Figure $1^{a}$

\begin{tabular}{cccccccc}
\hline $\begin{array}{c}c \\
(\mathrm{~g} / \mathrm{mL})\end{array}$ & $\begin{array}{c}n \\
\left(\mathrm{~nm}^{-3}\right)\end{array}$ & $\begin{array}{c}\rho_{\text {solv }} \\
\left(\mathrm{nm}^{-2}\right)\end{array}$ & $\begin{array}{c}\bar{a} \\
(\mathrm{~nm})\end{array}$ & $\begin{array}{c}\delta \\
(\mathrm{nm})\end{array}$ & $A$ & $\phi^{\mathrm{HS}}$ & $\alpha$ \\
\hline 0.0042 & $7.00 \times 10^{-8}$ & $6.257 \times 10^{-4}$ & 22.5 & 3.0 & 0.20 & & \\
0.0481 & $9.00 \times 10^{-7}$ & $6.241 \times 10^{-4}$ & 22.5 & 3.0 & 0.20 & 0.067 & 1.0 \\
0.2109 & $4.53 \times 10^{-6}$ & $6.129 \times 10^{-4}$ & 22.5 & 3.0 & 0.20 & 0.34 & 1.0 \\
0.3042 & $6.53 \times 10^{-6}$ & $6.025 \times 10^{-4}$ & 22.5 & 3.0 & 0.20 & 0.45 & 0.98 \\
0.3266 & $7.00 \times 10^{-6}$ & $5.996 \times 10^{-4}$ & 22.5 & 3.0 & 0.20 & 0.47 & 0.97 \\
0.3539 & $7.60 \times 10^{-6}$ & $5.958 \times 10^{-4}$ & 22.5 & 3.0 & 0.20 & 0.51 & 0.97 \\
0.3989 & $8.56 \times 10^{-6}$ & $5.889 \times 10^{-4}$ & 22.5 & 3.0 & 0.20 & 0.56 & 0.96
\end{tabular}

$a$ The scattering length density of the polystyrene cores was fixed to $1.0 \times 10^{-4} \mathrm{~nm}^{-2}$. Incomplete solvent exchange in stock dispersions results in a variation of $\rho_{\text {solv }}$ with particle concentration. The $\rho_{\text {solv }}$ values provided here have been calculated from the measured solvent composition in the stock dispersions.

proceeded at $70 \pm 0.1{ }^{\circ} \mathrm{C}$ for $24 \mathrm{~h}$. A nitrogen atmosphere was supplied throughout the reactions.

After cooling to room temperature, the raw product was filtered in two steps, first by gravity ( 1001 filter paper, Munktell) followed by $0.8 / 0.45 \mu \mathrm{m}$ membrane filtration (Pall, Gelman Laboratory). Purification by medium exchange was achieved by diafiltration (a technique not only very effective in removing unwanted species but also time saving ${ }^{53}$ ) for the $\mathrm{H} 8$ latex and centrifugal filtration for the D8 latex. The purity was monitored by conductivity $(\kappa)$ until the value of pure solvent was reached $(\kappa \approx 1.0 \mu \mathrm{S} / \mathrm{cm}$, for the H8 latex, and $4.3 \mu \mathrm{S} / \mathrm{cm}$, for latex D8). Diafiltration and centrifugal filtration against the solvent with $10 \mathrm{mM}$ added electrolyte $\left(3.85 \mathrm{mM} \mathrm{NaN}_{3}\right.$ and $\left.6.15 \mathrm{mM} \mathrm{NaCl}, \kappa \approx 0.9 \mathrm{mS} / \mathrm{cm}\right)$ was also monitored by conductivity. Addition of a small amount of salt to the solvent served two purposes: to screen residual surface charges (mainly $-\mathrm{SO}_{4}{ }^{2-}$ ) originating from the initiator, confirmed by electrophoresis, and, in addition, to prevent bacterial growth in the latices during longer-time storage. The final step in preparation of both latex H8 and D8 was obtaining concentrated dispersions, achieved by centrifugal filtration of stock dispersions to sufficiently high mass content.

Exchange of $\mathrm{H}_{2} \mathrm{O}$ for $\mathrm{D}_{2} \mathrm{O}$ as the solvent for latex $\mathrm{H} 8$ was done using repeated centrifugal filtration, diluting concentrated dispersions with $\mathrm{D}_{2} \mathrm{O}$ after each cycle. Progress was monitored by measuring the density of the filtrate. In the final concentrated stock dispersion, some residual $\mathrm{H}_{2} \mathrm{O}$ causes a slight variation in solvent composition upon constructing a concentration series by diluting with $\mathrm{D}_{2} \mathrm{O}$. Solvent scattering length densities have been calculated taking this effect into account (cf. Table 2).

Dynamic light scattering (DLS) measurements were done using an ALV CGS-8F DLS/SLS-5022F instrument (ALV, Langen, Germany), equipped with an ALV-6010/160 correlator and dual APD detectors, at a wavelength of $632.8 \mathrm{~nm}$. The scattering angle and temperature were kept constant at 90 and $25.0{ }^{\circ} \mathrm{C}$, respectively. Size distributions were extracted using a CONTIN analysis $^{54,55}$ from 10 averaged measurements of diluted dispersions. Care was taken to ensure that size distributions were invariant upon further sample dilution.

The density of the core-shell particles was extracted from a linear least-squares slope of the reciprocal density as a function of weight fraction. Here, inherently an average property of the composite particles is obtained with contributions from the partial densities of both core and layer. The particle density at $25.00{ }^{\circ} \mathrm{C}$ was determined as $1.0757 \mathrm{~g} / \mathrm{cm}^{3}$ for latex H8 and $1.1509 \mathrm{~g} / \mathrm{cm}^{3}$ for D8 using a high-precision density meter (DMA5000, AntonPaar). Particle densities were used to determine solvent volumes, required for preparing sodium carbonate containing samples of specified ionic strength at arbitrary particle concentrations. The weight fractions $(X)$ of stock solutions were determined from drying at $60^{\circ} \mathrm{C}$ until stable values were reached. The five highest concentrations in the dilution series of the $\mathrm{H} 8$ latex were prepared from one stock solution $(X=0.4079)$, whereas the remaining two 543.

(53) Labib, M. E.; Robertson, A. A. J. Colloid Interface Sci. 1978, 67

(54) Provencher, S. W. Comput. Phys. Comm. 1982, 27, 213.

(55) Provencher, S. W. Comput. Phys. Comm. 1982, 27, 229. (most dilute) samples originated from another stock solution $(X$ $=0.2847)$. The D8 latex samples were all prepared by diluting from one stock solution $(X=0.1999)$.

SANS experiments were performed at the large scale structure diffractometer D22 at the Institut Laue Langevin (Grenoble, France). Three sample-to-detector distances (1.5, 8.0, and 17.6 $\mathrm{m})$ together with three collimation lengths $(4.0,8.0$, and $17.6 \mathrm{~m})$ resulted in a $q$ range, $0.002<q<0.5 \AA^{-1}$, for $\lambda=7 \AA$ used in all measurements. All measurements were done using rectangular quartz cuvettes from Helma with a 1 or $2 \mathrm{~mm}$ optical path length, together with a thermostated sample holder held at a constant temperature of $25 \pm 0.1^{\circ} \mathrm{C}$. Two-dimensional spectra were azimuthally averaged using standard programs. After background subtraction, correction for empty cell, and transmissions, absolute intensities were determined by using water as a standard. Data sets from different sample-to-detector distances overlapped without adjustment of scale.

When modeling experimentally obtained SANS data, theoretical model predictions should be smeared to account for limitations in the instrumental setup, which tend to smooth out otherwise sharp features in the measured data. As summarized concisely in ref 46 , we make use of the analytical expressions given by Pedersen et al. ${ }^{56}$ of the resolution function, approximated by a Gaussian function, including in this way contributions from finite collimation, detector resolution, and wavelength spread $(\Delta \lambda / \lambda)$. The tails of the Gaussian function were truncated after three standard deviations, following essentially work by Barker and Pedersen; 57 with the instrumental settings used here, the resolution function was found to be insensitive to the precise value of the Gaussian tail cutoff. The finite collimation effect on the resolution of the scalar $q$ vector originates from the work of Mildner and Carpenter, who derived expressions for both circular and rectangular geometries. ${ }^{58}$

As input to the smearing algorithm, we supply collimation distances, detector resolution (full-width at half-maximum), 0.8 $\mathrm{cm}$, detector annulus width, $1 \mathrm{~cm}, \Delta \lambda / \lambda=0.10$, source aperture dimensions, $4.0 \times 5.5 \mathrm{~cm}$, and sample aperture dimensions, 0.7 $\times 1.0 \mathrm{~cm}$. Additional smearing effects, such as beam-stop shadowing and gravity effects, are neglected.

\section{Results and Discussion}

We have conducted a SANS study of core-shell colloids with grafted PEG, covering a large range of particle concentrations and two different solvent conditions for PEG. For particles with protonated cores, substitution of $\mathrm{H}_{2} \mathrm{O}$ for $\mathrm{D}_{2} \mathrm{O}$ enhances the particle contrast and reduces incoherent scattering, which is used here to focus on the effect of particle concentration. Because the PEG layer is poorly resolved in this choice of contrast, contrast variation on particles with nearly completely deuterated cores (D8) is used to shift focus to the structural properties of the PEG layer. This is done both under good and marginal solvent conditions, as regulated by the addition of sodium carbonate.

Scattering under Good Solvent Conditions. Beginning with stable dispersions under good solvent conditions ( $\left[\mathrm{Na}_{2} \mathrm{CO}_{3}\right]=0 \mathrm{M}$ and $25{ }^{\circ} \mathrm{C}$ ), we have investigated how the scattering evolves on increasing the particle concentration and the extent to which an effective hardsphere model captures particle correlations. Water is an excellent solvent for free PEG at room temperature.59 Changing the solvent to $\mathrm{D}_{2} \mathrm{O}$ alters the solubility of PEG somewhat ${ }^{60,61}$ and lowers the cloud point curve of PEG

(56) Pedersen, J. S.; Posselt, D.; Mortensen, K. J. Appl. Crystallogr. 1990, 23, 321 .

(57) Barker, J. G.; Pedersen, J. S. J. Appl. Crystallogr. 1995, 28, 105

(58) Mildner, D. F. R.; Carpenter, J. M. J. Appl. Crystallogr. 1984, 17,249

(59) Saeki, S.; Kuwahara, N.; Nakata, M.; Kaneko, M. Polymer 1976 17,685 .

(60) Branca, C.; Faraone, A.; Maisano, G.; Magazú, S.; Migliardo, P.; Triolo, A.; Triolo, R.; Villari, V. J. Phys.: Condens. Matter 1999, 11, 6079 . 


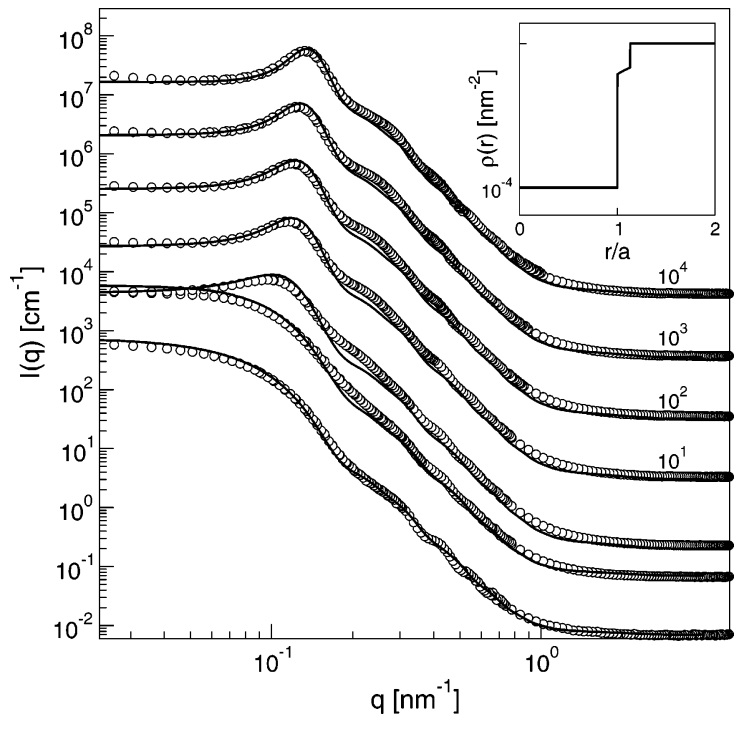

Figure 1. Scattered intensity as a function of $q$ and the mass concentration, from top to bottom, $c=0.40,0.35,0.33,0.30$, $0.21,0.048$, and $0.0042 \mathrm{~g} / \mathrm{mL}$. The highest concentrations have been shifted for clarity, with the shift factors given along the right-hand side. The lines are fits of eq 1 to the data, using eq 7 for the form factor together with the Percus-Yevick solution for polydisperse hard spheres. The inset shows a typical particle contrast profile as a function of the normalized radial distance from the particle center for latex H8, here for $c=0.0042 \mathrm{~g} / \mathrm{mL}$.

solutions by $\approx 10{ }^{\circ} \mathrm{C} .{ }^{62}$ However, in the absence of extra added sodium carbonate, the cloud point remains located at high temperatures, and this substitution is of no consequence here.

The scattered intensity for latex H8 is shown in Figure 1 as a function of $q$ and particle concentration together with fits of the model. The form factor is determined using the expression in eq 7 and is compared with the most dilute scattering curve, where structure factor contributions are vanishingly small. In all cases here, the contrast of the shell is close to that of the solvent (see inset of Figure 1). As a consequence, we find that the fits are rather insensitive to the parameters governing the solvated PEG shell. In fact, the most dilute scattering curve can be reasonably well-fitted by neglecting the shell altogether, i.e., by modeling the scattering as that from a collection of homogeneous cores. Clearly, to obtain any detailed information about the shell, contrast conditions closer to core matching are required. However, the fits at high $q$ are somewhat improved by including a shell of thickness $3 \mathrm{~nm}$ with a PEG surface coverage of $20 \%$.

The treatment of polydispersity in our modeling effort requires several comments. Electron microscopy on dried dispersions was unsuccessful. Particles lose their identity either partially or completely because of coalescence. As an alternative to electron microscopy, CONTIN analy$\operatorname{ses}^{54,55}$ of DLS data on dilute samples were used to gain information on the particle size distribution. As shown by the representative example in Figure 2, the resulting size distributions were skewed such that few large particles are present. Using the size distributions obtained in this way directly in the SANS analysis yielded qualitatively reasonable fits with a substantial smearing of the first form factor minimum but leaving a clear structure in the high $q$ part of the scattering curves. This trend is

(61) Branca, C.; Faraone, A.; Magazú, S.; Maisano, G.; Migliardo, P.; Triolo, A.; Triolo, R.; Villari, V. J. Appl. Crystallogr. 2000, 33, 709.

(62) Thiyagarajan, P.; Chaiko, D. J.; Hjelm, R. P. Macromolecules 1995, 28,7730

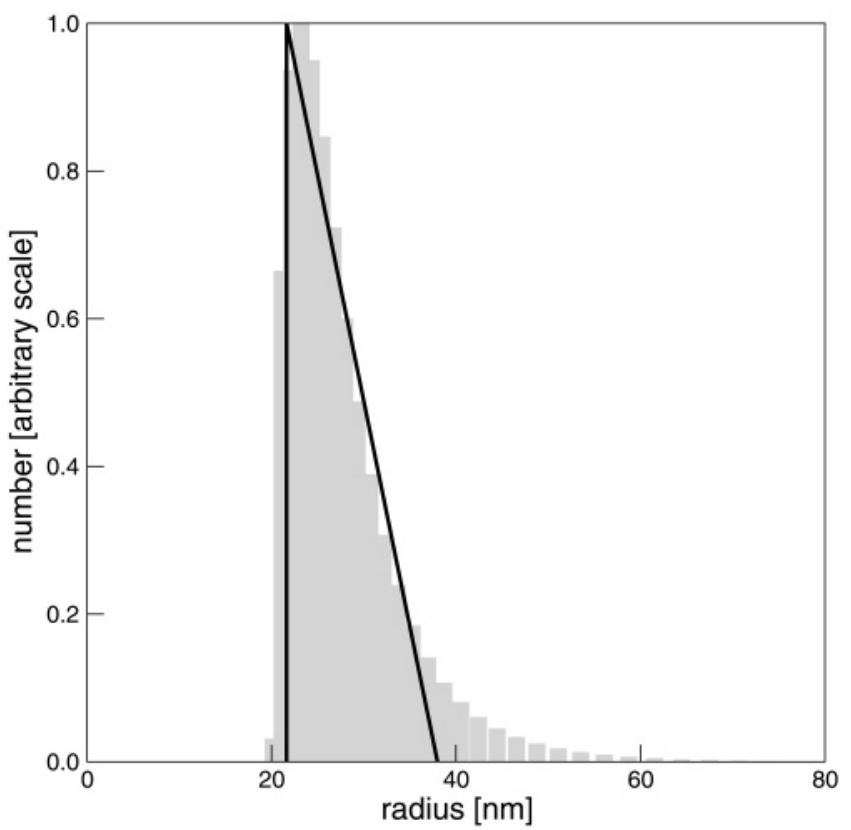

Figure 2. Typical size distribution obtained from a CONTIN analysis of DLS on dilute dispersions from latex H8. The solid line shows the triangular size distribution used in the SANS modeling.

particularly evident for the most dilute sample in Figure 1 , where a smeared first minimum, followed by three resolved oscillations, is observed. As discussed by Pontoni et al. ${ }^{63}$ and as verified separately in this study, standard distributions (Gauss, log normal, and Schulz) do not retain a high $q$ structure on introducing a sufficient polydispersity to smear the first form factor minimum to the extent that we observe. Instead, skewed distributions, rich in small particles, are required. Unfortunately, the polydispersities predicted by using the CONTIN-produced size distributions are too large, overestimating the degree of smearing in the form factor minima, particularly the first. This is not too surprising; while CONTIN is a stable and reliable program, its smoothing algorithm affects the ability to resolve narrow size distributions correctly. ${ }^{64}$ We have chosen a skewed triangular distribution (Figure 2) because it is close to the size distributions obtained by DLS when we truncate them before the tail with large particles sets in. Having imposed a size distribution, the size polydispersity is determined almost entirely by the degree of smearing of the form factor minima. The fitted polydispersity for latex $\mathrm{H} 8$, in terms of the normalized standard deviation, is $16.5 \%$.

Once the parameters of the form factor are determined, taking dilution of stock dispersions into account in determining sample concentrations, our model only allows for one additional parameter in capturing structure factor effects, the parameter $\alpha$. It serves to reduce the hardsphere interaction radii from their nominal values, $a_{i}+$ $\delta$, which somewhat crudely is meant to capture a softer, repulsive interaction, stemming from interpenetration or compression of PEG layers. Proceeding with the modeling of the remainder of the concentration series in Figure 1 accordingly, we observe two discrepancies that need to be

(63) Pontoni, D.; Finet, S.; Narayanan, T.; Rennie, A. R. J. Chem. Phys. 2003, 119, 6157.

(64) Schurtenberger, P.; Newman, M. E. Characterization of biologica and environmental particles using static and dynamic light scattering. In Characterization of Environmental Particles; Buffle, J., van Leeuwen, H. P., Eds.; IUPAC Environmental Analytical Chemistry Series; Lewis Publishers: Boca Raton, FL, 1993; Vol. 2. 
addressed. First, the absolute intensities of the two most dilute samples do not scale with the dilution factors used. We attribute this error to their having been prepared from a different stock dispersion than the rest of the samples shown in Figure 1. No such mismatch in absolute intensity was observed in other samples (not shown) diluted down to similar concentrations from another H8 stock dispersion. Thus, the two most dilute samples are not shown on an absolute scale; they have been shifted vertically along the logarithmic axis in Figure 1 to comply with the dilution series from the more concentrated stock dispersion. Second, a simultaneous fitting of all of the scattering curves, i.e., using the same form factor parameters, requires the scattering length density of the core to be set to $\rho_{\text {core }}=1.0 \times 10^{-4} \mathrm{~nm}^{-2}$. This value, compared to the literature values for polystyrene, $\rho_{\mathrm{ps}}=1.4 \times 10^{-4} \mathrm{~nm}^{-2}$, and PEG, $\rho_{\text {peg }}=6.37 \times 10^{-5} \mathrm{~nm}^{-2}$, suggests either that some PEG is present in what we define as the core or that the transition from the core to shell is far more gradual than what we propose in eq 5 . Smoothing of the coreshell interface in eq 5 has been included in modeling similarly structured particles. ${ }^{29,36,41,43}$ Instead of opting for a more complex scattering length density profile, which inevitably introduces more adjustable parameters, we retain a homogeneous core but with $\rho_{\text {core }}=1.0 \times 10^{-4}$ $\mathrm{nm}^{-2}$, because this is sufficient to obtain reasonably accurate fits to the scattering curves over the whole concentration range.

Upon increasing the particle concentration, several effects are observed. Excluded-volume interactions lead to positional correlations, such that the low $q$ intensity is suppressed and a peak emerges at intermediate $q$. The effective hard-sphere model captures this effect semiquantitatively provided that the hard-sphere radii are somewhat reduced (proportionally, via $\alpha$ ) for concentrations above $c=0.21 \mathrm{~g} / \mathrm{mL}$. At the highest concentration, a $4 \%$ reduction of the hard-sphere radii corresponds roughly to a $1 \mathrm{~nm}$ compression of PEG layers. While reasonable, the result is of course subject to errors incurred from not having used the correct interaction potential. The complete set of parameters used in the fits is reported in Table 2.

As seen in Figure 1, there are significant changes in the scattering curves in the region of the first form factor minimum in going from the dilute samples to the more concentrated ones. In Figure 3, we have enhanced the intermediate $q$ region, where this effect is present. Here, we observe that, when scaling with the particle concentration, the scattering curves for latex $\mathrm{H} 8$ (top panel of Figure 3) do not superpose in this region. A tentative explanation, quite plausible for particles with grafted, polymer layers, is that the form factor is dependent upon the particle concentration. It is well-known that the radius of gyration of free PEG in good solvents decreases as a function of the concentration below the dilute-semidilute crossover concentration. ${ }^{62}$ Also, swollen core-shell, microgel particles have been observed to exhibit a concentration-dependent form factor. ${ }^{65}$ It stands to reason that such changes in concentration should derive from structural changes of the PEG layer.

To probe the structure of the PEG layer, it is necessary to use contrast variation. To this end, we have synthesized PEG-grafted particles with nearly completely deuterated polystyrene cores dispersed in $\mathrm{D}_{2} \mathrm{O}$, referred to as latex D8. Not only does deuteration lead to a substantial reduction of incoherent scattering, but it also shifts the

(65) Stieger, M.; Pedersen, J. S.; Lindner, P.; Richtering, W. Langmuir 2004, 20, 7283 .

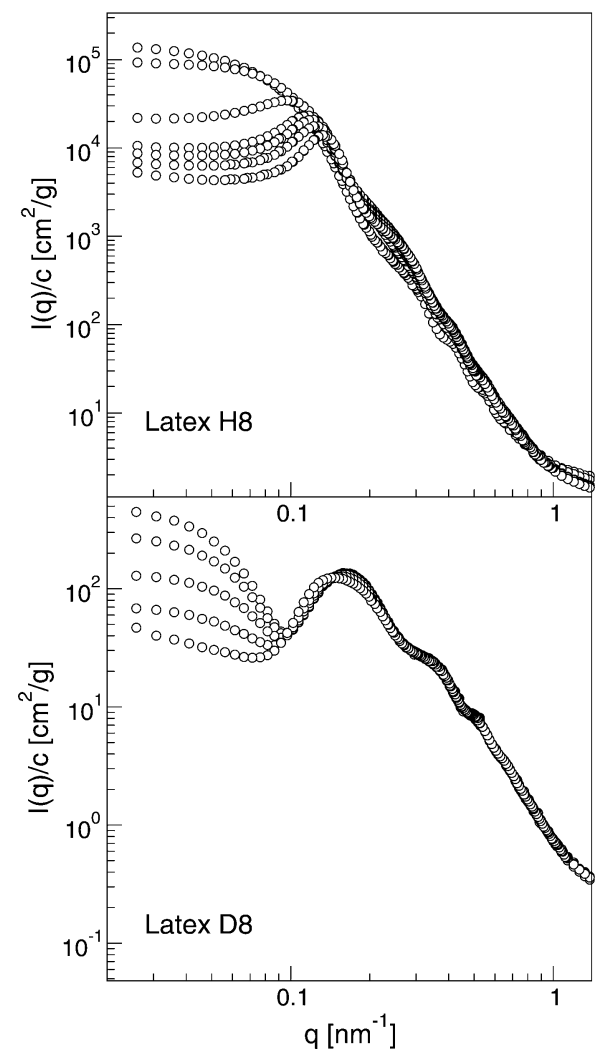

Figure 3. Scattered intensity, normalized by mass concentration $c$ of particles, for latex H8 (top panel) and latex D8 (lower panel) as a function of $q$ and $c$. As described in the text, the two data sets have been obtained under two very different contrast conditions (cf. insets to Figures 1 and 7). Concentrations are the same as in Figures 1 and 6.

scattering length density of the core close to that of the solvent such that the comparatively weak scattering from the solvated PEG shell is revealed.

Because we have incorporated some protonated styrene in the particle cores (to make sure that under all circumstances $\rho_{\mathrm{H}_{2} \mathrm{O}}<\rho_{\text {core }}<\rho_{\mathrm{D}_{2} \mathrm{O}}$ ) and some residual PEG may be incorporated in the cores, we do not have a sufficiently accurate estimate of $\rho_{\text {core }}$ to determine $a$ priori the solvent composition corresponding to core matching (shell scattering). In cases such as this, in the context of SANS, contrast variation is the method of choice. There are however some complications that need to be considered. The minimum intensity, in the limit $q \rightarrow 0$, is obtained when the volume average of the contrast profile vanishes (particle matching). Instead of this condition, we would prefer to reach core matching, but this contrast produces more scattering in the $q \rightarrow 0$ limit. Nevertheless, because contrast variation has to be done on site, a reasonable compromise is to seek for the minimum intensity because this should yield scattering that is quite sensitive to the properties of the PEG layer. For weakly scattering samples near matching conditions, obtaining data close to $q=0$ is however exceedingly time-consuming. However, given that we are not interested in precise particle matching, we varied the solvent contrast, through variation of the weight ratio $\mathrm{D}_{2} \mathrm{O} / \mathrm{H}_{2} \mathrm{O}$, to identify conditions for minimum scattering in a window of finite $q, 0.057<q<1.15 \mathrm{~nm}^{-1}$.

In Figure 4, we show averaged intensity data for latex D8 as a function of the solvent composition, both in the absence and presence of added $\mathrm{Na}_{2} \mathrm{CO}_{3}$. As seen, the presence of extra salt (above the $10 \mathrm{mM}$ background electrolyte present in all samples) shifts the solvent composition for minimum scattering somewhat. The 


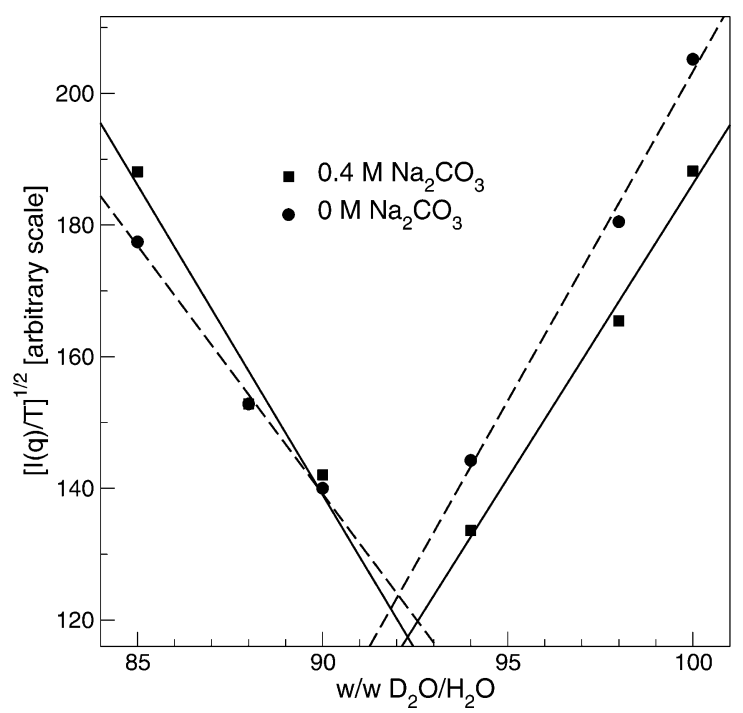

Figure 4. Square root of the intensity (not on an absolute scale), averaged over the $q$ interval $0.057<q<1.15 \mathrm{~nm}^{-1}$ and normalized by transmission, as a function of the solvent composition, as labeled. Lines are guides to the eye.

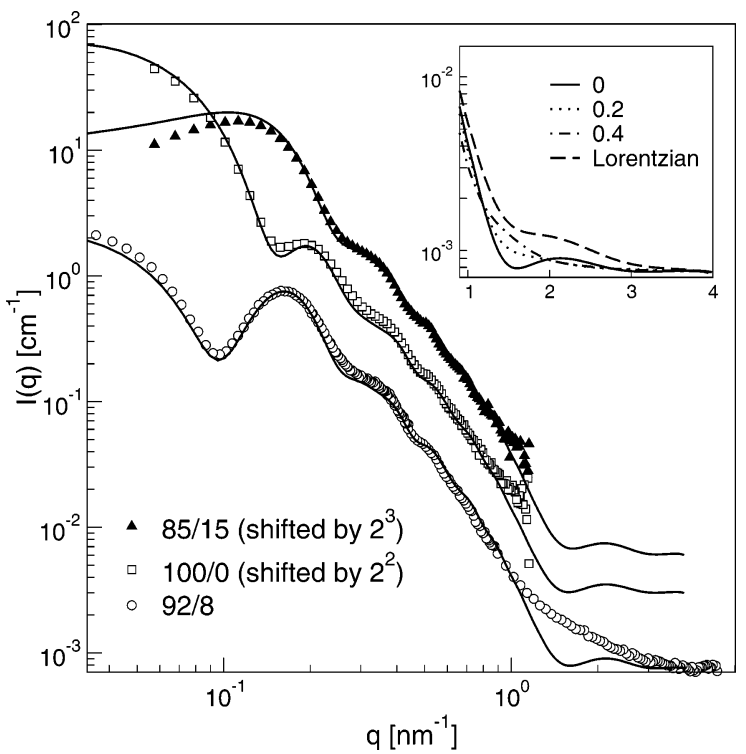

Figure 5. Intensity as a function of $q$ for three different contrasts close to the core and overall particle matchpoints [100:0, 92:8, and 85:15 (w/w) $\left.\mathrm{D}_{2} \mathrm{O} / \mathrm{H}_{2} \mathrm{O}\right]$ at a fixed particle concentration, $c=0.0056 \mathrm{~g} / \mathrm{mL}$, for latex D8. The data have been vertically shifted as labeled. Lines are form factor fits of eq 7 to the data in which only the calculated $\rho_{\text {solv }}$ values vary. The inset shows qualitative effects on the model at high $q$ if 0 , 20 , and $40 \%$ polydispersity in shell thickness $\delta$ is introduced and when a Lorentz function is added.

solvent compositions corresponding to minimum scattering in Figure 4 were selected for preparation of dilution series extending up to particle concentrations of roughly $c=0.2 \mathrm{~g} / \mathrm{mL}$.

For a more reliable quantitative analysis, we have also determined full scattering curves under dilute conditions for three contrasts, two of which bracket the one corresponding to the minimum scattering in Figure 4. These are shown in Figure 5. Data were fitted simultaneously, meaning that among the three data sets in Figure 5 only the calculated $\rho_{\text {solv }}$ was allowed to vary according to the known solvent composition. As seen in the inset to Figure 7 , the contrast conditions corresponding to the scattering curves in Figure 5 span roughly core matching [ 100:0 (w/w) $\mathrm{D}_{2} \mathrm{O} / \mathrm{H}_{2} \mathrm{O}$ ] to slightly past particle matching. Even though the data go through dramatic changes from one contrast condition to the other, the model is able to follow closely. As opposed to the analysis of the H8 samples, the fits are sensitive to the parameters connected with the PEG layer. A somewhat thicker, $\delta=4 \mathrm{~nm}$, more dense shell, $A=0.29$, is required to fit the data. Also, the particle core radius is somewhat smaller compared to latex $\mathrm{H} 8$, $20.5 \mathrm{~nm}$, and the polydispersity is lower, $13.5 \%$, differences that may be a result of the two different syntheses.

The PEG concentration in the shell, determined from eq 4 , is $18 \mathrm{vol} \%$, similar to what has been observed using other syntheses. ${ }^{66}$ In setting up the form amplitude model, a polymer density profile was obtained by assuming fully stretched chains. However, the shell thickness that we obtain is only about one-third of the contour length. ${ }^{67} \mathrm{We}$ rationalize this result as follows. The lower scattering length density value $\rho_{\text {core }}$, compared to the nominal polystyrene value, suggests that not all of the PEG is present at the particle periphery; rather, some of the PEG chain length, when modeled with a step-function-like profile, may end up residing in what we define as the core. In other words, the polymer density profile is very likely more complex than what is assumed in eq 3. For instance, for planar PEG brushes in $\mathrm{D}_{2} \mathrm{O}, \delta$ values around $5 \mathrm{~nm}$ have been obtained for $10^{4} \mathrm{~g} / \mathrm{mol} \mathrm{PEG}$, but with an additional low-density tail in the profile extending to larger distances. ${ }^{68}$ Such a tail, if present, would be exceedingly difficult to detect with SANS on spherical particles.

As seen in Figure 5, significant deviations between the model and data set in at high $q$. Here, we probe small length scales, comparable to or smaller than the layer thickness. It is a characteristic feature of simple coreshell models, such as ours, that, upon approaching small length scales, shell interference leads to oscillations of a period in $q$ of $\sim 2 \pi \delta^{-1}$; it is the first minimum of such an oscillation that is seen in the model at $q \approx 1.6 \mathrm{~nm}^{-1}$ in Figure 5. No such oscillation is observed in the experimental data. When the layer thickness or the PEG coverage is reduced, this high $q$ structure in the model is shifted beyond the experimentally accessible $q$ range. However, it is important to emphasize that, in contrast to the scattering data from latex $\mathrm{H} 8$, the data in Figure 5 demand thick, fairly dense layers when considering all but the highest $q$; improving the fits at high $q$ by decreasing $\delta$ or $A$ completely spoils the overall agreement with the data. Hence, it is far more likely that some effect is present that smears the large $q$ oscillations. The model, on the basis of homogeneous, monodisperse PEG layers, evidently provides insufficient resolution to capture the smoothing out of these oscillations. In the inset of Figure 5, we supply some ways of extending the model. Introducing layer polydispersity, uncoupled to the polydispersity in core size, can be done easily for a layer thickness distributed according to a flat, top-hat distribution. As seen, layer polydispersity tends to gradually smooth out the sharper features of the basic model. Following others, ${ }^{42,69}$ we can also improve the agreement at high $q$ by appealing to polymer fluctuations and superpose a Lorentzian factor, $I_{\text {fluct }}(0) / 1+(q \xi)^{2}$, describing average correlations in a polymer network. More sophisticated approaches to handling high $q$ structures have also been suggested. ${ }^{70}$ In what follows, we will accept the shortcomings at high $q$ and

(66) Drobek, T.; Spencer, N. D.; Heuberger, M. Macromolecules 2005 , 38,5254 .

(67) Oesterhelt, F.; Rief, M.; Gaub, H. E. New J. Phys. 1999, 1, 6.1.

(68) Irvine, D. J.; Myers, A. M.; Satija, S. K.; Barker, J. G.; Sofia-

Allgor, S. J.; Griffiths, L. G. J. Biomed. Mater. Res. 1998, 40, 498

(69) Dingenouts, N.; Norhausen, Ch.; Ballauff, M. Macromolecules 1998, 31, 8912.

(70) Pedersen, J. S. J. Chem. Phys. 2001, 114, 2839. 


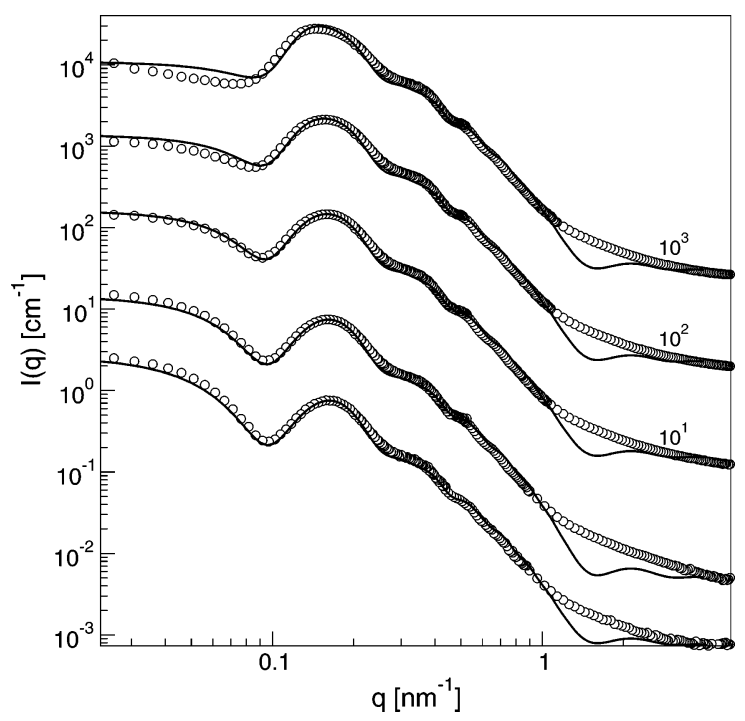

Figure 6. Intensity as a function of $q$ for latex $\mathrm{D} 8$ concentration series, shown with model fits corresponding to the parameters in Table 3. The data correspond to particle concentrations, from top to bottom, $c=0.2213,0.1660,0.1107,0.0553$, and 0.0055 $\mathrm{g} / \mathrm{mL}$. Data have been offset for clarity with shift factors given along the right-hand side.

proceed with the basic model, also keeping the number of adjustable parameters at a minimum.

In Figure 6, we show scattering curves for a dilution series, $0.0055 \leq c \leq 0.22 \mathrm{~g} / \mathrm{mL}$, at contrast conditions corresponding to minimum scattering from Figure 4, i.e., 92:8 (w/w) $\mathrm{D}_{2} \mathrm{O} / \mathrm{H}_{2} \mathrm{O}$. The form factor parameters determined from the most dilute sample (Figure 5 ) have been kept fixed in the modeling of these results. Structure factor effects are handled as before, with an effective hard-sphere model incorporating just one additional adjustable parameter. Given this, the fits to the data are quite good, and as for the H8 latex in the corresponding concentration range, the best fits are obtained by using $\alpha=1$. In Figure 3 (lower panel), the same data have been scaled by the particle concentration, highlighting the rather extreme suppression of scattering at low $q$ together with the subtle changes in the peak region at intermediate $q$. At the higher concentrations, the primary peak of the structure factor begins to become noticeable, entering at $q$ values just to the left of the form factor peak at $q \approx 0.16 \mathrm{~nm}^{-1}$. Surprisingly, the concentration scaling in Figure 3 perfectly superposes the scattering data for all of the concentrations at $q$ larger than the first form factor peak; in this region, structure factor effects are negligible. This result is in contrast to what was observed in Figure 1 (cf. also top panel of Figure 3) and indeed what in part motivated this contrast-variation study. A tentative explanation was given in the context of Figure 1 as concentration effects on the form factor, possibly caused by layer contraction and densification. This appears now very unlikely in light of the result in Figure 3. Rather, we find no evidence of variation in PEG layer properties on going from dilute to more concentrated systems, at least under this contrast, which is far more sensitive to properties of the PEG layer. We can also rule out isotope effects associated with $\mathrm{D}_{2} \mathrm{O} / \mathrm{H}_{2} \mathrm{O}$ exchange through SAXS measurements (not shown), where form factors for latex D8 superpose perfectly in very different solvent compositions. We are unable to find a plausible explanation for this difference between latex H8 and D8 in Figure 3, although it may be that the different degrees of incoherent

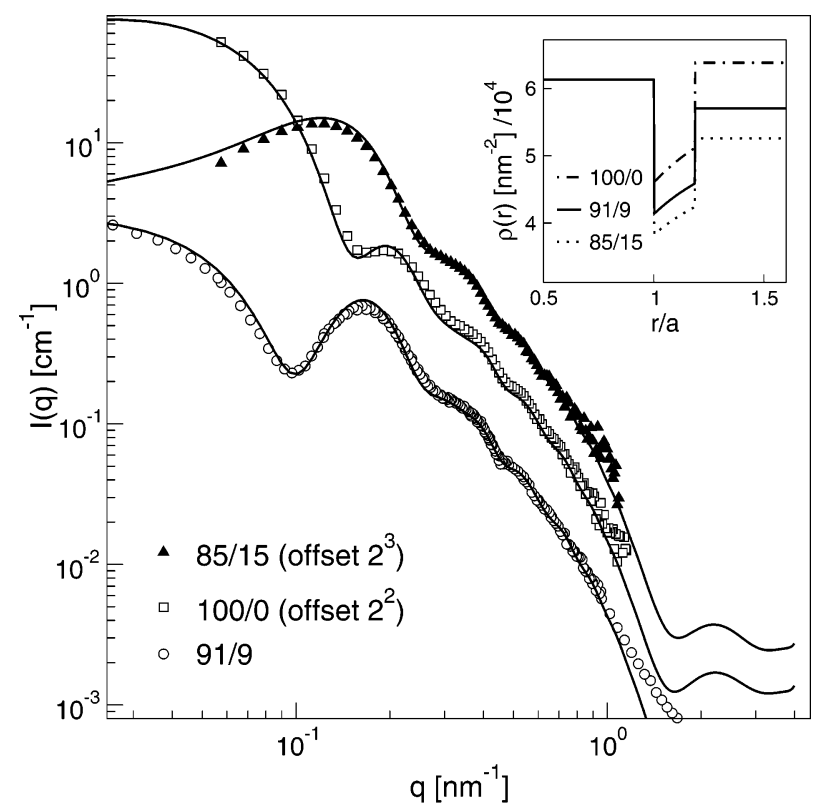

Figure 7. Form factors measured for latex D8 in $0.4 \mathrm{M} \mathrm{Na}_{2} \mathrm{CO}_{3}$ at three different contrasts corresponding to 100:0, 91:9, and $85: 15(\mathrm{w} / \mathrm{w}) \mathrm{D}_{2} \mathrm{O} / \mathrm{H}_{2} \mathrm{O}$. Lines are "simultaneous" fits of eq 7 in which only the calculated $\rho_{\text {solv }}$ values vary. Shown in the inset are accompanying scattering length density profiles (cf. eq 5), as labeled.

scattering under the different contrast conditions can produce such effects. ${ }^{71}$

Scattering under Marginal Solvent Conditions. Adding $\mathrm{Na}_{2} \mathrm{CO}_{3}$ produces divalent $\mathrm{CO}_{3}{ }^{-2}$ ions in aqueous solution $(\mathrm{pH} \sim 11.5)$, which worsens the solvent quality for PEG chains. In solutions of free PEG, the cloud point curve, above which PEG solutions phase separate in dilute and semidilute coexisting phases, is substantially lowered ${ }^{72,73}$ upon addition of $\mathrm{Na}_{2} \mathrm{CO}_{3}$. The precise clouding temperature depends upon the PEG molecular weight and solution composition. Roughly, however, adding a few hundred millimolar $\mathrm{Na}_{2} \mathrm{CO}_{3}$ brings clouding temperatures down in the vicinity of room temperature for low molecular weight PEG. Our latices remain stable at all concentrations in solutions of $0.4 \mathrm{M} \mathrm{Na}_{2} \mathrm{CO}_{3}$ at $25{ }^{\circ} \mathrm{C}$. However, upon raising the temperature slightly above $25^{\circ} \mathrm{C}$, the particles aggregate reversibly; more precisely, samples of $c \approx 0.2 \mathrm{~g} / \mathrm{mL}$ become unstable at around $T \approx 26-27^{\circ} \mathrm{C}$, whereas the more dilute samples remain stable up to somewhat higher temperatures.

Optimal contrast for dispersions in $0.4 \mathrm{M} \mathrm{Na}_{2} \mathrm{CO}_{3}$ was determined as 91:9 (w/w) $\mathrm{D}_{2} \mathrm{O} / \mathrm{H}_{2} \mathrm{O}$ from the minimum in average intensity, shown in Figure 4, which is slightly shifted compared to the $0 \mathrm{M}$ case. In Figure 7, form factors measured at three contrasts, 100:0, 91:9, and 85:15 (w/w) $\mathrm{D}_{2} \mathrm{O} / \mathrm{H}_{2} \mathrm{O}$, are reported. Shown also are simultaneous fits of the core-shell model (eq 7). As before, only the calculated scattering length densities of the solvents vary according to the known solvent compositions, with all other parameters being subject to the constraint of not varying with the contrast in Figure 7. From fits of the core-shell model, which provides a good description of the form factor data in Figure 7, we find a small contraction and densification of the PEG layer, $\delta=3.85 \mathrm{~nm}$ and $A=0.31$, compared to results obtained in the absence of $\mathrm{Na}_{2} \mathrm{CO}_{3}$.

(71) Rosenfeldt, S.; Dingenouts, N.; Ballauff, M.; Lindner, P.; Likos, C. N.; Werner, N.; Vögtle, F. Macromol. Chem. Phys. 2002, 203, 1995.

(72) Boucher, E. A.; Hines, P. M. J. Polym. Sci. 1976, 14, 2241.

(73) Taboada, M. E.; Asenjo, J. A.; Andrews, B. A. Fluid Phase Equilib. 2001, 180, 273. 


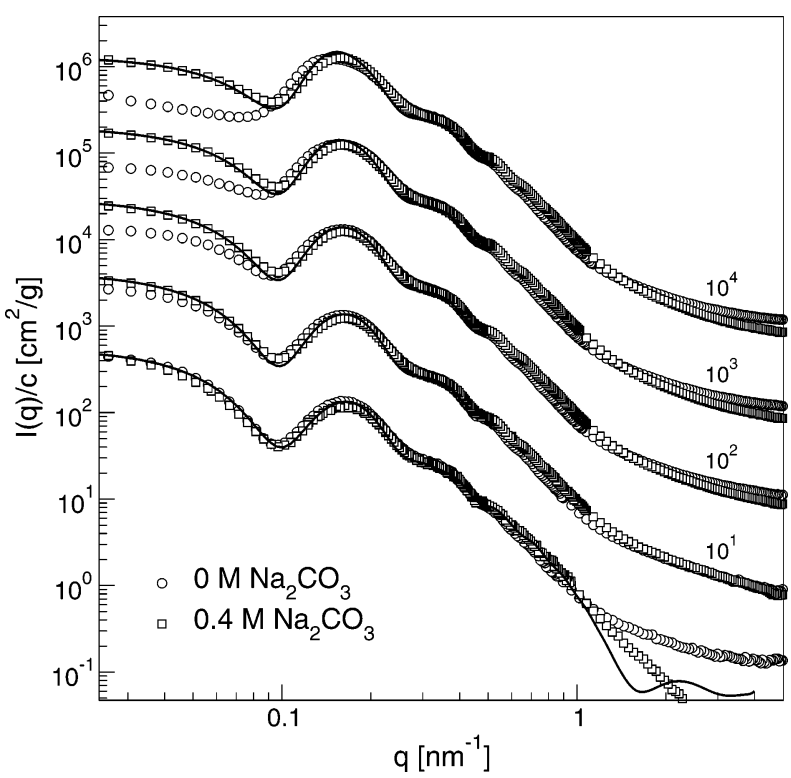

Figure 8. Scattered intensity as a function of $q$ for the concentration series of latex D8, both with and without $0.4 \mathrm{M}$ $\mathrm{Na}_{2} \mathrm{CO}_{3}$, at $91 / 9$ and $92 / 8 \mathrm{w} / \mathrm{w} \mathrm{D}_{2} \mathrm{O} / \mathrm{H}_{2} \mathrm{O}$, respectively. Data have been normalized by particle concentration and shifted for clarity, as labeled along the right hand side. Solid lines at low-tointermediate $q$ are fits of Percus-Yevick solutions for squarewell interactions (eq 8).

Roughly, only a $5 \%$ change in these parameters occur upon adding the extra salt. Note that the core radius and polydispersity do not vary from the values determined without added $\mathrm{Na}_{2} \mathrm{CO}_{3}$.

Although we detect only small structural changes upon adding salt, the particle interactions are significantly altered, going from close to hard-sphere-like to attractive. This is seen in Figure 8, where the scattering data from the concentration series of latex D8 with $\left[\mathrm{Na}_{2} \mathrm{CO}_{3}\right]=0.4$ $\mathrm{M}$, covering $0.0057 \leq c \leq 0.2275 \mathrm{~g} / \mathrm{mL}$, is compared with data in the absence of $\mathrm{Na}_{2} \mathrm{CO}_{3}$. Drastic differences are observed at low $q$, where scattering from the dispersions in the presence of salt is not nearly as suppressed. Slight differences in the peak shape are also seen at the higher concentrations. We attribute these differences to the emergence of attractions among particles when $\mathrm{Na}_{2} \mathrm{CO}_{3}$ is added. The low $q$ behavior of the scattered intensity together with the fact that particles aggregate at somewhat higher temperatures is firm support for this.

To model the presence of attractions, we have opted for a full numerical solution of the Ornstein-Zernike equation with the Percus-Yevick closure for a square-well attraction. The explicit form of the square-well attraction is

$$
u_{i j}(r)= \begin{cases}\infty & 0<r<\sigma_{i j}^{\mathrm{HS}} \\ -\epsilon & \sigma_{i j}^{\mathrm{HS}}<r<R \sigma_{i j}^{\mathrm{HS}} \\ 0 & R \sigma_{i j}^{\mathrm{HS}}<r\end{cases}
$$

where $r$ is the separation distance, $\sigma_{i j}^{\mathrm{HS}}=a_{i}^{\mathrm{HS}}+a_{j}^{\mathrm{HS}}$ is the minimum separation distance between particles belonging to species $i$ and $j, \epsilon$ is the well depth, and $R$ is related to the range over which attractions act. Following D'Aguanno and Klein, ${ }^{44}$ this has been done for a $N=3$ component representation of the continuous, triangular size distribution (see Appendix A). This number of components, however, is too low to obtain a good model at high $q$, where the discrete nature of the distribution is revealed through unphysical oscillations in the model. As a consequence, fits are only shown from low up to intermediate $q$, where, after all, structure factor effects are operative; the model
Table 3. Model Parameter Values Obtained from Fitting the Effective Hard-Sphere Model to the $I(q)$ Data from the Concentration Series of Latex D8 in 92:8 (w/w) $\mathrm{D}_{2} \mathrm{O} / \mathrm{H}_{2} \mathrm{O}$, Corresponding to the Solid Lines in Figure $6^{a}$

\begin{tabular}{ccccccc}
\hline \multicolumn{7}{c}{$\left[\mathrm{Na}_{2} \mathrm{CO}_{3}\right]=0 \mathrm{M}$} \\
$c(\mathrm{~g} / \mathrm{mL})$ & $n\left(\mathrm{~nm}^{-3}\right)$ & $\bar{a}(\mathrm{~nm})$ & $\delta(\mathrm{nm})$ & $A$ & $\phi^{\mathrm{HS}}$ & $\alpha$ \\
\hline 0.0056 & $1.20 \times 10^{-7}$ & 20.5 & 4.0 & 0.29 & & \\
0.0557 & $1.20 \times 10^{-6}$ & 20.5 & 4.0 & 0.29 & 0.077 & 1.0 \\
0.1114 & $2.38 \times 10^{-6}$ & 20.5 & 4.0 & 0.29 & 0.154 & 1.0 \\
0.1671 & $3.58 \times 10^{-6}$ & 20.5 & 4.0 & 0.29 & 0.230 & 1.0 \\
0.2228 & $4.78 \times 10^{-6}$ & 20.5 & 4.0 & 0.29 & 0.230 & 1.0 \\
\multicolumn{7}{c}{$\left[\mathrm{Na}_{2} \mathrm{CO}_{3}\right]=0.4 \mathrm{M}$} \\
$c(\mathrm{~g} / \mathrm{mL})$ & $n\left(\mathrm{~nm}^{-3}\right)$ & $\bar{a}(\mathrm{~nm})$ & $\delta(\mathrm{nm})$ & $A$ & $\phi^{\mathrm{HS}}$ & $B_{2} / B_{2}^{\mathrm{HS}}$ \\
\hline 0.0057 & $1.20 \times 10^{-7}$ & 20.5 & 3.85 & 0.31 & & \\
0.0569 & $1.20 \times 10^{-6}$ & 20.5 & 3.85 & 0.31 & 0.077 & 0.47 \\
0.1137 & $2.39 \times 10^{-6}$ & 20.5 & 3.85 & 0.31 & 0.153 & 0.47 \\
0.1706 & $3.59 \times 10^{-6}$ & 20.5 & 3.85 & 0.31 & 0.229 & 0.47 \\
0.2275 & $4.79 \times 10^{-6}$ & 20.5 & 3.85 & 0.31 & 0.306 & 0.47
\end{tabular}

${ }^{a}$ The lower part of the table lists the corresponding results for latex $\mathrm{D} 8$, dispersed in $0.4 \mathrm{M} \mathrm{Na}_{2} \mathrm{CO}_{3}$ and $91: 9(\mathrm{w} / \mathrm{w}) \mathrm{D}_{2} \mathrm{O} / \mathrm{H}_{2} \mathrm{O}$, with the resulting fits shown in Figures 7 and 8 . Here, the model was supplemented with attractive interactions characterized by a reduced second virial coefficient $B_{2} / B_{2}^{\mathrm{HS}}$ ( $\alpha=1$ was used for all concentrations).

results merge with the form factor fit, also shown in Figure 8 , obtained for the most dilute sample at intermediate $q$. The square-well attraction introduces a range and depth, which cannot be identified separately in a meaningful way from comparisons with the data that we have available. As a compromise, we have chosen to set $R=$ 1.04 and report the well depth via a simplified second virial coefficient, given by

$$
B_{2} / B_{2}^{\mathrm{HS}}=1-\left(R^{3}-1\right)\left(e^{\epsilon / k T}-1\right)
$$

As a result, with the form factor parameters kept constant and taking dilution factors into account, the remainder of the concentration series is modeled with just one parameter, $\epsilon / k T$.

The resulting fits are shown in Figure 8, and the corresponding parameter values are reported in Table 3. Both the evolution of the low $q$ scattering and the scattering around the peak at intermediate $q$ with the concentration are well-modeled by introducing moderate attractions. The second virial coefficient is found to be independent of the concentration and is reduced by $\sim 50 \%$ upon adding $0.4 \mathrm{M} \mathrm{Na}_{2} \mathrm{CO}_{3}$, corresponding to a well depth of $1.65 k T$ for $R=1.04$. Evidently, what appears to be small structural changes in the PEG layer results in attractions of measurable magnitude. Conceivably, the PEG layer undergoes more dramatic changes on a molecular scale ${ }^{74}$ beyond the resolution of our SANS experiments.

In view of the discrepancies between model fits and scattering data at high $q$, we proceed to buttress our assertions that (i) the PEG layer changes are slight but measurable upon addition of $\mathrm{Na}_{2} \mathrm{CO}_{3}$ and (ii) that no form factor changes occur upon increasing the particle concentration. To this end, we show all of the scattering curves for latex D8, both with and without $\mathrm{Na}_{2} \mathrm{CO}_{3}$, in Figure 9 . Here, they are shown in a Porod plot, $q^{4} I(q)$, scaled by the particle concentration, highlighting the subtle changes at intermediate-to-high $q$ upon introducing $\mathrm{Na}_{2} \mathrm{CO}_{3}$. As seen, the data superpose along two curves, one corresponding to added $\mathrm{Na}_{2} \mathrm{CO}_{3}$ and the other without. Note that there are no detectable changes in this representation

(74) Roke, S.; van Blaaderen, A.; Bonn, M. Manuscript to be published. 


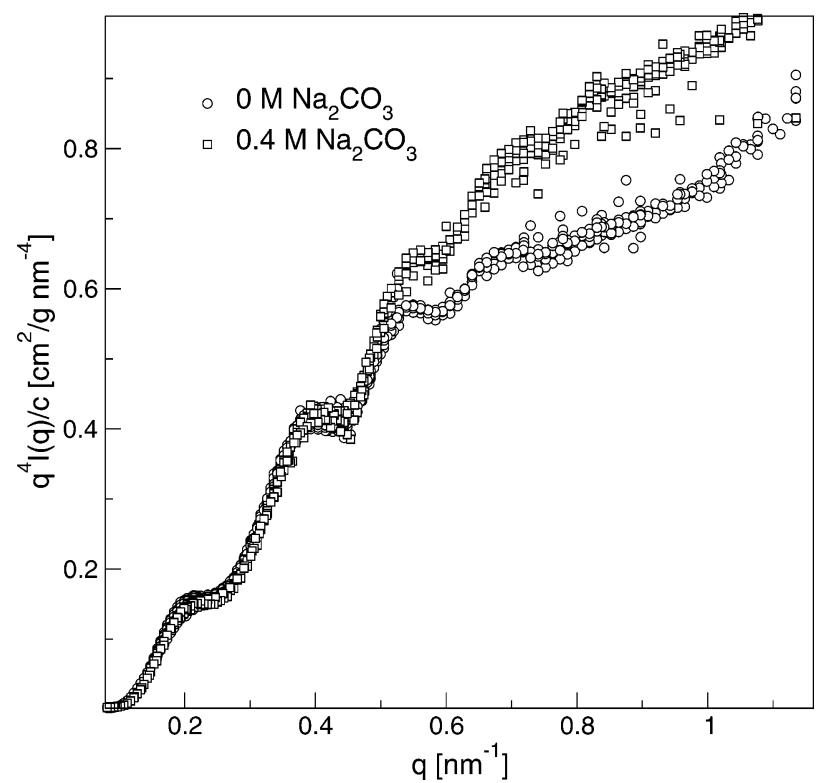

Figure 9. Porod plot of scattering curves for latex D8, both with and without $0.4 \mathrm{M} \mathrm{Na}_{2} \mathrm{CO}_{3}$, in $91: 9$ and $92: 8$ (w/w) $\mathrm{D}_{2} \mathrm{O} /$ $\mathrm{H}_{2} \mathrm{O}$, respectively. All concentrations (cf. Table 3) are shown.

within each concentration series, demonstrating that the polymer layer does not change with the particle concentration.

Much work has been done in the past on solvent quality effects on steric interactions. ${ }^{2}$ Continuous collapse of polymer layers upon diminishing solvent quality is a welldocumented effect. ${ }^{42,69,75}$ We observe no such drastic reduction in PEG layer thickness at $\left[\mathrm{Na}_{2} \mathrm{CO}_{3}\right]=0.4 \mathrm{M}$, close to aggregation, although it may set in when the solvent quality is further decreased. Particle attractions, however, appear before structural changes of such a magnitude occur. The emergence of attractions upon reducing the solvent quality has been observed among ethylene-oxide-based block copolymer midelles ${ }^{76}$ and adsorbed surfactants. ${ }^{77}$ In the latter case, however, they are associated with an increase in the layer thickness. Presumably, the grafting density plays an important role, ${ }^{2}$ such that low surface coverages and poor solvent conditions favor chain interpenetration and some extension, whereas high surface coverages prevent this from occurring.

\section{Conclusions}

Three concentration series of PEG-grafted polystyrene particles have been examined with SANS under varying neutron contrast and solvent quality. Quantitative analysis is expedited by modeling the particles as core-shell colloids. An effective hard-sphere model captures interactions in a good solvent up to high concentrations; however, at the highest concentrations, departure from true hardsphere behavior is observed, suggesting a softer repulsion. Resolving the PEG layer requires drastically changing the contrast. Contrast variation, through isotopic exchange of both the core and solvent, shifts focus from the overall, composite particle to the PEG layer, which has been studied both in the dilute limit and as a function of the particle concentration. Contrary to what can be inferred from particle contrast conditions, no change in PEG layer

(75) Karim, A.; Satija, S. K.; Douglas, J. F.; Ankner, J. F.; Fetters, L. J. Phys. Rev. Lett. 1994, 73, 3407.

(76) Liu, Y.; Chen, S.-H.; Huang, J. S. Macromolecules 1998, 31, 2236.

(77) Claesson, P. M.; Kjellander, R.; Stenius, P.; Christenson, H. K. J. Chem. Soc., Faraday Trans. 1 1986, 82, 2735. properties is detected as a function of the particle concentration, up to moderate concentrations. Upon diminishing the solvent quality, subtle changes in the PEG layer translate into attractions among particles. Modeling the additional attractions with a one-parameter representation of the square-well interaction, we find that they are of moderate magnitude. As will be reported in forthcoming work, the magnitude of these interactions vary smoothly with the solvent quality such that particles can be made to aggregate in a fully reversible fashion. Also, as found here, the particles are moderately polydisperse, enough to preclude crystallization at high concentrations. This can be exploited in studying the dynamics and rheology upon approaching the glassy state directly from the single-phase fluid, as will be reported elsewhere.

\section{Appendix A: Size Distribution}

In this work, the size distribution is approximated by a triangular distribution skewed such that few large particles are present. Having assigned a continuous size distribution $F(a)$ to our system, we immediately face the problem that calculations of form and structure factors are more readily done for discrete size distributions. We follow the methodology developed by D'Aguanno and Klein $^{44}$ and approximate the continuous distribution by a discrete one. They replace the continuous distribution with an effective $N$-component mixture, by requiring equality of moments. Application of this method results in a set of $2 N$-coupled, nonlinear equations, which is difficult to solve unless $N$ is small. Often a small number of components suffices, but for modeling scattering data extending to high $q, N$ needs to be fairly large, prompting us to look for an alternative procedure. We require that the $n$th moment be matched between continuous and discrete representations as

$$
X^{n}=\int_{a_{0}}^{a_{1}} d a a^{n} F(a)=\sum_{i=1}^{N} x_{i} a_{i}^{n}
$$

where $\bar{a}$ will denote the mean radius, and for the triangular distribution

$$
F(a)=\frac{a_{1}-a}{9 \sigma_{a}^{2}} \text { for } a_{0} \leq a \leq a_{1}
$$

where $a_{0}=\bar{a}-\sqrt{2} \sigma_{a}, a_{1}=\bar{a}+2 \sqrt{2} \sigma_{a}$, and $\sigma_{a}$ is the standard deviation of the size or radius distribution. The normalized, continuous size distribution is fully determined by the first and second moments. The higher order moments are easily calculated as

$$
\begin{gathered}
\frac{X^{(n)}}{\left(X^{(1)}\right)^{n}}=2 \times 3^{n} \frac{(1-\kappa)^{-2}}{(1+2 \kappa)^{n}}\left[\frac{1}{(n+1)(n+2)}+\right. \\
\left.\kappa^{n+1\left(\frac{\kappa}{n+2}-\frac{1}{n+1}\right)}\right]
\end{gathered}
$$

where $\kappa=a_{0} / a_{1}$. For this particular choice of size distribution, we can achieve exact matching of the first $2 \mathrm{~N}$ moments of the continuous and discrete size distributions by making a change of variable as $z=1-2\left[\left(a_{1}-\right.\right.$ $\left.a) /\left(a_{1}-a_{0}\right)\right]$. Substitution in eq 10 yields then a standard integral for application of Gauss-Jacobi quadrature 


$$
\begin{array}{r}
X^{(n)}=\frac{\left(a_{1}-a_{0}\right)^{2}}{36 \sigma_{a}^{2}} \int_{-1}^{1} \mathrm{~d} z\left(a_{1}-\frac{(1-z)\left(a_{1}-a_{0}\right)}{2}\right)^{n} \times \\
(1-z) \approx \sum_{i=1}^{N} x_{i} a_{i}^{n}
\end{array}
$$

where $x_{i}=\left[\left(a_{1}-a_{0}\right)^{2} /\left(36 \sigma_{a}^{2}\right)\right] w_{i}$ and $a_{i}=a_{1}-\left[(1-z)\left(a_{1}\right.\right.$ $\left.\left.-a_{0}\right)\right] / 2$ are mole fractions and radii of the histogram in terms of $w_{i}$ and $z_{i}$, the Gauss-Jacobi weights and abscissas, respectively. The weights and abscissas were determined numerically ${ }^{78}$ for a prescribed number of components, $N$, of the histogram representation of the continuous polydisperse system. We have used $N=40$ in

(78) Press, W. H.; Teukolsky, S. A.; Vetterling, W. T.; Flannery, B. P. Numerical Recipes in FORTRAN, 2nd ed.; Cambridge University Press: New York, 1992. our modeling. This procedure is not limited to triangular size distributions; it can easily be adapted for Schulz $(\Gamma)$ distributed sizes using Gauss-Laguerre quadrature.

Acknowledgment. Financial support from the Swedish Foundation for Strategic Research (SSF, Program for Colloid and Interface Technology) and the Swedish Research Council is gratefully acknowledged. This work was further supported by the State Secretariat for Education and Research (SBF Nb. 03.0566), the Marie Curie Network on Dynamical Arrest of Soft Matter and Colloids (MRTN-CT-2003-504712), and the Swiss National Science Foundation. J. B. is a Royal Swedish Academy of Sciences Research Fellow, supported by a grant from the Knut and Alice Wallenberg Foundation. We are grateful to the Institut Laue Langevin for beam time and Ralf Schweins for his help and expertise.

LA051664V 